\title{
Entraves e benefícios na utilização do ensino remoto para os acadêmicos do curso de enfermagem durante a pandemia de COVID-19: revisão integrativa
}

Obstacles and benefits in the use of remote education for students in the nursing course during the COVID-19 pandemic: integrative review

Obstáculos y beneficios en el uso de educación a distancia para estudiantes del curso de enfermería durante la pandémica COVID-19: revisión integradora

Jaqueline Brito da Costa ORCID: https://orcid.org/0000-0002-2570-1714 Centro Universitário de Ciências e Tecnologia do Maranhão, Brasil E-mail: Jaquelinebritto2012@gmail.com Karine Costa Melo ORCID: https://orcid.org/0000-0001-8253-859X

Universidade Federal do Maranhão, Brasil Email: karinemelo09@gmail.com

Jairina Nunes Chaves ORCID: https://orcid.org/0000-0002-3547-6901 Universidade Estadual do Maranhão, Brasil E-mail: inanunes@hotmail.com

Marconny Lira da Silva

ORCID: https://orcid.org/0000-0003-0334-9294 Centro Tecnológico de Ensino Múltiplo, Brasil E-mail: marconnylirads@hotmail.com

Laine da Costa Almeida Barboza

ORCID: https://orcid.org/0000-0002-9801-1546 Centro Universitário de Ciências e Tecnologia do Maranhão, Brasil E-mail: laynnealmeidah@gmail.com

Paulo Vicente Dourado

ORCID: https://orcid.org/0000-0002-0101-3423 Faculdade Malta, Brasil

E-mail: Paulo.vdourado@yahoo.com.br

Linccon Fricks Hernandes

ORCID: https://orcid.org/0000-0002-7642-3080 Escola Superior de Ciências da Santa Casa de Misericórdia de Vitória, Brasil

E-mail: fricksjr@hotmail.com

Núbia Oliveira da Silva

ORCID: https://orcid.org/0000-0002-6544-7415

Universidade Estadual do Maranhão, Brasil E-mail: nubiasilva.enf@hotmail.com

Ana Carla Marques da Costa

ORCID: https://orcid.org/0000-0002-4246-145X Centro Universitário de Ciências e Tecnologia do Maranhão, Brasil E-mail: carlama271@gmail.com

Márcia Sousa Santos

ORCID: https://orcid.org/0000-0002-6517-0479 Centro Universitário de Ciências e Tecnologia do Maranhão, Brasil E-mail: mssenfermeira@gmail.com

Camilla Lohanny Azevedo Viana ORCID: https://orcid.org/0000-0002-4529-3607 Centro Universitário de Ciências e Tecnologia do Maranhão, Brasil E-mail: camillalohanny@hotmail.com

Francisco das Chagas Araújo Sousa ORCID: https://orcid.org/0000-0001-7244-9729 Universidade Estadual do Piauí, Brasil E-mail: franciscoaraujo@ccs.uespi.br

Hálmisson D'Árley Santos Siqueira ORCID: https://orcid.org/0000-0001-9831-5892 Centro Universitário de Ciências e Tecnologia do Maranhão, Brasil E-mail: halmisson@yahoo.com.br 


\author{
Hádila Giovanna Santos Siqueira Cunha \\ ORCID: https://orcid.org/0000-0002-8401-6119 \\ Universidade Federal do Maranhão, Brasil \\ E-mail: hadilagiovanna@ hotmail.com \\ Francisco Braz Milanez Oliveira \\ ORCID: https://orcid.org/0000-0003-3841-0104 \\ Centro Universitário de Ciências e Tecnologia do Maranhão, Brasil \\ E-mail: braz_cm@hotmail.com
}

\begin{abstract}
Resumo
A Pandemia de COVID-19 ocasionou diversos impactos ao redor do mundo e em março de 2020, devido a velocidade que o vírus se espalhou pelos continentes, para tentar conter o avanço foi criada a política de distanciamento social, responsável pela interrupção de inúmeras atividades, incluindo aulas presenciais. Assim, ampliou-se a busca por meios de ensino remoto, a fim de amenizar os prejuízos causados na educação. $O$ estudo tem como objetivo geral analisar os entraves na utilização do ensino remoto para os acadêmicos de enfermagem durante a pandemia de COVID-19. Trata-se de uma pesquisa bibliográfica do tipo revisão integrativa da literatura. Sete artigos foram incluídos nesta revisão, a maior parte apresentou abordagem qualitativa dos dados $(71,4 \%)$. A base de dados CINAHL forneceu o maior número de estudos $(71,4 \%)$. Todos os artigos concentraram-se no ano de 2021 e estavam na língua inglesa (100\%); em que os Estados Unidos e Nepal foram os países com o maior número de publicações, com 28,6\% cada. O periódico Nurse Education Today forneceu o maior número de evidências (28,6\%). Com relação ao delineamento de pesquisa todos os estudos eram transversais (100\%), onde todos representavam nível de evidência seis. A pandemia afetou a forma como o conhecimento é repassado, e dificultou a realização de aulas práticas para os alunos onde, o ensino remoto se tornou a alternativa mais segura e eficaz, utilizando diversas plataformas online, como Zoom, Google Meet e Teams.
\end{abstract}

Palavras-chave: Estudantes de enfermagem; Educação a distância; COVID-19.

\begin{abstract}
The COVID-19 Pandemic caused several impacts around the world and in March 2020, due to the speed at which the virus spread across the continents, the social distancing policy was created to try to contain the advance, responsible for the interruption of numerous activities, including face-to-face classes. Thus, the search for remote teaching means was expanded, in order to alleviate the damage caused in education. The study has as general objective to analyze the obstacles in the use of remote learning for nursing students during the COVID-19 pandemic. This is bibliographic research of the integrative literature review type. Seven articles were included in this review, most of which presented a qualitative approach to data $(71.4 \%)$. The CINAHL database provided the largest number of studies (71.4\%). All articles focused on the year 2021 and were in English (100\%); in which the United States and Nepal were the countries with the highest number of publications, with $28.6 \%$ each. The journal Nurse Education Today provided the greatest amount of evidence (28.6\%). Regarding the research design, all studies were cross-sectional (100\%), where all represented evidence level six. The pandemic affected the way knowledge is transferred, and made it difficult for students to carry out practical classes, where remote learning has become the safest and most effective alternative, using various online platforms, such as Zoom, Google Meet and Teams.
\end{abstract}

Keywords: Students, nursing; Education distance; COVID-19.

\title{
Resumen
}

La Pandemia COVID-19 causó varios impactos en todo el mundo y en marzo de 2020, debido a la velocidad a la que el virus se extendió por los continentes, se creó la política de distanciamiento social para tratar de contener el avance, responsable de la interrupción de numerosas actividades. incluyendo clases presenciales. Así, se amplió la búsqueda de medios de enseñanza a distancia, con el fin de paliar el daño causado en la educación. El estudio tiene como objetivo analizar los obstáculos en el uso del aprendizaje a distancia para estudiantes de enfermería durante la pandemia COVID-19. Se trata de una investigación bibliográfica del tipo revisión integradora de la literatura. En esta revisión se incluyeron siete artículos, la mayoría de los cuales presentaban un enfoque cualitativo de los datos (71,4\%). La base de datos CINAHL proporcionó el mayor número de estudios (71,4\%). Todos los artículos se centraron en el año 2021 y estaban en inglés (100\%); en el que Estados Unidos y Nepal fueron los países con mayor número de publicaciones, con un 28,6\% cada uno. La revista Nurse Education Today proporcionó la mayor cantidad de evidencia (28,6\%). En cuanto al diseño de la investigación, todos los estudios fueron transversales (100\%), donde todos representaron el nivel de evidencia seis. La pandemia afectó la forma en que se transfieren los conocimientos y dificultó la realización de las clases prácticas por parte de los alumnos, donde el aprendizaje remoto se ha convertido en la alternativa más segura y eficaz, utilizando diversas plataformas online, como Zoom, Google Meet y Teams.

Palabras clave: Estudiantes de enfermería; Educación a distancia; COVID-19. 


\section{Introdução}

A Pandemia de COVID-19 afetou gravemente o funcionamento normal dos serviços de saúde, como os centros de atenção primária e hospitais, sobrecarregando o sistema de saúde (SUS). Com recursos limitados, o governo precisou fazer ampliação de leitos hospitalares, compras de medicamentos, equipamentos, ações para fornecer um melhor atendimento aos pacientes em estado graves (Sarti et al., 2020).

Em março de 2020 devido a velocidade que o vírus se espalhou pelos continentes, para tentar conter o avanço foi criada a política de distanciamento social, responsável pela interrupção de inúmeras atividades, incluindo aulas presenciais. Assim, ampliou-se a busca por meios de ensino remoto, a fim de amenizar os prejuízos causados na educação (Magalhães et al., 2020).

Neste contexto, as instituições de ensino presencial se deparam com a necessidade de adquirir os recursos tecnológicos, que até então eram domínio dos cursos online, híbridos e a distância, para dar continuidade ao seu processo educacional mediantes as tecnologias (Bueno et al., 2020).

As consequências da pandemia o setor educacional impulsionou um processo de adequação da formação acadêmica, para isso foram feitos novos planos de aulas emergenciais nas universidades, inclusive na área da saúde, ajustes no desenvolvimento institucional, projetos pedagógicos de cursos de graduações e no gerenciamento departamental para fazer frente à situação de emergência (Gusso et al., 2020).

O estudo tem como problema de investigação, quais as dificuldades e benefícios apresentados na modalidade de ensino remoto para os acadêmicos de enfermagem durante a pandemia de COVID-19? Para tal como objetivo geral analisar os entraves na utilização do ensino remoto para os acadêmicos de enfermagem durante a pandemia de COVID-19. E como objetivos específicos investigar os benefícios advindos do ensino remoto para os acadêmicos frente ao contexto de pandemia e identificar os recursos tecnológicos utilizados durante as aulas de ensino remoto.

O ensino remoto é uma ferramenta que possibilita através da tecnologia a adequação à necessidade do contexto de pandemia causada pela COVID-19, permite a continuidade da conclusão das grades curriculares. Assim, a temática torna-se relevante, pois aborda a importância do ensino remoto para os acadêmicos de enfermagem, possibilitando a sua permaneçam durante todo o tempo da pandemia, no qual as aulas remotas foram estratégias necessárias e essenciais para o repasse dos conteúdos acadêmicos, capacitando os discentes no uso de novas tecnologias no sistema educacional, sendo uma abordagem na readequação do aprendizado.

\section{Metodologia}

O presente estudo trata-se de uma pesquisa bibliográfica do tipo revisão integrativa da literatura. Este procedimento foi escolhido por possibilitar a síntese e análise do conhecimento científico já produzido sobre o tema "Entraves e benefícios na utilização do ensino remoto para os acadêmicos do curso de enfermagem durante a pandemia de COVID-19: revisão integrativa". Esta revisão utilizou a metodologia proposta no estudo de Oliveira et al. (2016).

O tema determinou a construção da estratégia PICo, que representa um acrônimo para População ou Problema (P), Interesse (I) e Contexto (Co), na qual foi utilizada para a geração da questão norteadora desta revisão integrativa da literatura: “Quais os entraves e benefícios apresentados na modalidade de ensino remoto para os acadêmicos do curso de enfermagem durante a pandemia de COVID-19?"

Para a localização dos estudos relevantes, que respondessem à pergunta de pesquisa, utilizou-se de descritores indexados e não indexados (palavras-chave) nos idiomas português, inglês e espanhol. Os descritores foram obtidos a partir do Medical Subject Headings (MESH), dos Descritores em Ciências da Saúde (DeCS) e dos títulos da Cumulative Index to Nursing and Allied Health Literature (CINAHL), como mostra o Quadro 1. 
Consultou-se por meio de descritores e palavras-chave as bases de dados PubMed da National Library of Medicine; Biblioteca Virtual da Saúde (BVS) coordenada pelo Centro Latino-Americano e do Caribe de Informação em Ciências da Saúde (BIREME) e composta de bases de dados bibliográficas produzidas pela Rede BVS, como a Literatura LatinoAmericana e do Caribe em Ciências da Saúde (LILACS), além da base de dados Medline e outros tipos de fontes de informação; e CINAHL.

Quadro 1. Elementos da estratégia PICo, descritores e palavras-chave utilizados. Caxias, MA, Brasil, 2021.

\begin{tabular}{|c|c|c|c|c|c|}
\hline \multicolumn{2}{|r|}{ Elementos } & Mesh & Decs & Títulos CINAHL & Palavras-chave \\
\hline $\mathbf{P}$ & $\begin{array}{l}\text { Acadêmicos de } \\
\text { enfermagem }\end{array}$ & $\begin{array}{c}\text { "Students, Nursing" } \\
\text { "Nursing" }\end{array}$ & $\begin{array}{c}\text { "Estudantes de Enfermagem" } \\
\text { "Students, Nursing" } \\
\text { "Estudiantes de Enfermería" } \\
\text { Enfermagem } \\
\text { Nursing } \\
\text { Enfermería }\end{array}$ & $\begin{array}{l}\text { "Students, } \\
\text { Nursing" }\end{array}$ & $\begin{array}{c}\text { "Academics of Nursing" } \\
\text { Nursing }\end{array}$ \\
\hline I & $\begin{array}{l}\text { Educação a } \\
\text { distância }\end{array}$ & $\begin{array}{l}\text { "Education, } \\
\text { Distance" } \\
\text { "Education, } \\
\text { Nursing" }\end{array}$ & $\begin{array}{l}\text { "Educação à Distância” } \\
\text { "Education, Distance" } \\
\text { "Educación a Distancia" } \\
\text { "Educação em Enfermagem" } \\
\text { "Education, Nursing" } \\
\text { "Educación en Enfermeria" }\end{array}$ & $\begin{array}{l}\text { "Education, } \\
\text { Nursing" } \\
\text { "Education, Non- } \\
\text { Traditional" }\end{array}$ & $\begin{array}{l}\text { "Education, Distance” } \\
\text { "Remote teaching" } \\
\text { "Distance learning", } \\
\text { "Online Education", }\end{array}$ \\
\hline Co & COVID-19 & $\begin{array}{l}\text { COVID-19 } \\
\text { "Coronavirus } \\
\text { Infections" }\end{array}$ & $\begin{array}{c}\text { COVID-19 } \\
\text { "Infecções por Coronavirus", } \\
\text { "Coronavirus Infections" } \\
\text { "Infecciones por Coronavirus" }\end{array}$ & $\begin{array}{l}\text { "Coronavirus } \\
\text { Infections" }\end{array}$ & $\begin{array}{c}\text { COVID-19 } \\
\text { "COVID-19 Pandemic" }\end{array}$ \\
\hline
\end{tabular}

Fonte: Descritores e Títulos (2021).

Os termos utilizados durante a pesquisa foram classificados e combinados nos bancos de dados, resultando em estratégias específicas de cada base (Quadro 2).

Quadro 2. Estratégias de busca utilizadas nas bases de dados BIREME, PUBMED e CINAHL. Caxias, MA, Brasil, 2021.

\begin{tabular}{|c|c|c|c|c|}
\hline BASE DE DADOS & ESTRATÉGIA DE BUSCA &  & 宸 &  \\
\hline $\begin{array}{c}\text { BIREME } \\
\text { (descritores Decs) }\end{array}$ & $\begin{array}{l}\text { ("Students, Nursing" OR Nursing OR "Academics of Nursing") AND ("Education, Nursing" } \\
\text { OR "Education, Distance" OR "Remote teaching" OR "Distance learning" OR "Online } \\
\text { Education") AND ("Coronavirus Infections" OR COVID-19 OR "COVID-19 Pandemic") }\end{array}$ & 0 & 0 & 0 \\
\hline $\begin{array}{c}\text { PubMed } \\
\text { (descriptors MeSH) }\end{array}$ & $\begin{array}{l}\text { (("Students, Nursing" OR Nursing OR "Academics of Nursing") AND ("Education, Nursing" } \\
\text { OR "Education, Distance" OR "Remote teaching" OR "Distance learning" OR "Online } \\
\text { Education")) AND ("Coronavirus Infections" OR COVID-19 OR "COVID-19 Pandemic") }\end{array}$ & 388 & 348 & 02 \\
\hline $\begin{array}{l}\text { CINAHL } \\
\text { (CINAHL } \\
\text { Headings) }\end{array}$ & $\begin{array}{l}\text { ("Students, Nursing" OR “Academics of Nursing” OR Nursing) AND ("Education, Non- } \\
\text { Traditional" OR "Education, Nursing" OR "Education, Distance" OR "Remote teaching" OR } \\
\text { "Distance learning" OR "Online Education") AND ("Coronavirus Infections" OR COVID-19 } \\
\text { OR"COVID-19 Pandemic") }\end{array}$ & 575 & 270 & 05 \\
\hline
\end{tabular}

Fonte: Bases de dados (2021).

Como critérios de inclusão utilizaram-se estudos disponíveis em sua totalidade, publicados nos últimos três anos, de 2019 até 2021, nos idiomas Português, Espanhol e Inglês. Foram excluídos da busca inicial capítulos de livros, resumos, textos incompletos, teses, dissertações, monografias, relatos técnicos e outras formas de publicação que não fossem artigos científicos completos. 
A análise para seleção dos estudos foi realizada em duas fases. Na primeira, os estudos foram pré-selecionados segundo os critérios de inclusão e exclusão e de acordo com a estratégia de funcionamento e busca de cada base de dados.

$\mathrm{Na}$ base PUBMED, como busca total foram encontrados trezentos e oitenta e oito (388) estudos, aplicando na pesquisa o filtro que limita por texto completo dos últimos três anos com humanos, obteve-se trezentos e quarenta e oito (348) estudos, destes foram analisados títulos e resumos e teve como resultado final de dois (2) estudos. Na CINAHL foram obtidos quinhentos e setenta e cinco (575) estudos como busca geral, sendo que limitando a busca para artigos com texto completo realizado nos últimos três anos com humanos, obteve-se duzentos e setenta (270) estudos, sendo cinco (5) condizentes com a questão desta pesquisa após a análise dos títulos e resumos. Na base de dados da BVS não foram encontrados artigos científicos para análise dos resultados.

$\mathrm{Na}$ segunda fase os estudos foram analisados quanto ao potencial de participação no estudo, avaliando o atendimento à questão de pesquisa, bem como o tipo de investigação, objetivos, amostra, método, desfechos e conclusão, resultando em sete (7) artigos.

Posteriormente, foram analisadas as informações coletadas nos artigos científicos e criadas categorias analíticas que facilitou a ordenação e a sumarização de cada estudo. Essa categorização foi realizada de forma descritiva, indicando os dados mais relevantes para o estudo. A pesquisa levou em consideração os aspectos éticos da pesquisa quanto às citações dos estudos, respeitando a autoria das ideias, os conceitos e as definições presentes nos artigos incluídos na revisão.

Optou-se pela análise em forma estatística e de forma de texto, utilizando cálculos matemáticos e inferências, que serão apresentados em quadros e tabelas para facilitar a visualização e compreensão. As evidências científicas foram classificadas segundo os níveis e graus de recomendação propostos por Bork (2005).

\section{Resultados}

Essa fase é organizada em duas partes. A primeira está relacionada com a caracterização dos estudos, já a segunda, relaciona-se ao cumprimento do objetivo do estudo, que consiste em conhecer os entraves e benefícios na utilização do ensino remoto para os acadêmicos do curso de enfermagem durante a pandemia de COVID-19.

\section{Caracterização dos estudos}

Dos sete artigos incluídos nesta revisão, a maior parte apresentou abordagem qualitativa dos dados (71,4\%). A base de dados CINAHL forneceu o maior número de estudos (71,4\%). Todos os artigos concentraram-se no ano de 2021 e estavam na língua inglesa (100\%); em que os Estados Unidos e Nepal foram os países com o maior número de publicações, com 28,6\% cada. O periódico Nurse Education Today forneceu o maior número de evidências $(28,6 \%)$. Com relação ao delineamento de pesquisa todos os estudos eram transversais (100\%), onde todos representavam nível de evidência seis.

Uma atenção especial deve ser dada para o grau de recomendação, todos estudos apoiam o uso de aprendizagem online (100\%), embora seja menos eficaz que a convencional, uma vez que, disciplinas práticas não são facilmente transferíveis para o modo online. Desse modo, ações para melhorar o aprendizado online são essenciais para alcançar uma educação de mais qualidade e reduzir os desafios encontrados. Esses dados podem ser consultados na Tabela 1. 
Tabela 1. Análise descritiva das produções científicas acerca dos desafios e benefícios na utilização do ensino remoto para os acadêmicos do curso de enfermagem durante a pandemia de covid-19. Caxias, MA, Brasil, 2021.

\begin{tabular}{|c|c|c|}
\hline Variáveis & $\mathbf{N}$ & $\%$ \\
\hline \multicolumn{3}{|l|}{ Base de dados } \\
\hline CINAHL & 5 & 71,4 \\
\hline PUBMED & 2 & 28,6 \\
\hline BIREME & - & - \\
\hline \multicolumn{3}{|l|}{ Abordagem do estudo } \\
\hline Qualitativa & 5 & 71,4 \\
\hline Quantitativo & 2 & 28,6 \\
\hline \multicolumn{3}{|l|}{ Ano } \\
\hline \multicolumn{3}{|l|}{ Idiomas } \\
\hline Inglês & 7 & 100 \\
\hline \multicolumn{3}{|l|}{ País } \\
\hline Arábia Saudita & 1 & 14,3 \\
\hline Nepal & 2 & 28,6 \\
\hline Suécia & 1 & 14,3 \\
\hline Estados Unidos & 2 & 28,6 \\
\hline Jordânia & 1 & 14,3 \\
\hline Teaching and Learning in Nursing & 1 & 14,3 \\
\hline International Journal of Nursing Education & 1 & 14,3 \\
\hline BMC Nursing & 1 & 14,3 \\
\hline Nurse Education Today & 2 & 28,6 \\
\hline Nursing fórum na independent voice for nursing & 1 & 14,3 \\
\hline Plos One & 1 & 14,3 \\
\hline \multicolumn{3}{|l|}{ Delineamento de pesquisa } \\
\hline Estudo Transversal & 7 & 100,0 \\
\hline \multicolumn{3}{|l|}{ Classificação da evidência } \\
\hline Nível 06 & 7 & 100,0 \\
\hline \multicolumn{3}{|l|}{ Grau de recomendação } \\
\hline A & 7 & 100,0 \\
\hline
\end{tabular}

Fonte: Bases de dados (2021).

O Quadro 3 mostra a distribuição dos estudos segundo autores e anos de publicação, título; tipo de estudo; objetivo principal e perfil amostral. Os estudos versaram sobre as perspectivas de estudantes de enfermagem sobre a aprendizagem online, a satisfação desses alunos sobre esse método de ensino, retratando suas experiencias com relação ao uso de diferentes tecnologias e suas atitudes frente a prática do ensino remoto em meio a COVID-19. 
Research, Society and Development, v. 11, n. 1, e44911124883, 2022

(CC BY 4.0) | ISSN 2525-3409 | DOI: http://dx.doi.org/10.33448/rsd-v11i1.24883

Quadro 3. Publicações incluídas segundo o título do artigo, autor, objetivo principal e perfil amostral. Caxias, MA, Brasil, 2021. (N=7)

\begin{tabular}{|c|c|c|c|c|}
\hline $\begin{array}{l}\mathbf{N}^{\circ} \text { DE } \\
\text { ORDEM E } \\
\text { BASE }\end{array}$ & TÍTULO DO ARTIGO & AUTORES & OBJETIVO PRINCIPAL & PERFIL AMOSTRAL \\
\hline $\begin{array}{c}\text { A1 } \\
\text { CINAHL }\end{array}$ & $\begin{array}{l}\text { Nursing students' and faculty members' } \\
\text { perspectives about online learning during COVID- } \\
19 \text { pandemic: A qualitative study }\end{array}$ & Bdair (2021) & $\begin{array}{l}\text { Explorar as perspectivas de aprendizagem online de estudantes } \\
\text { e docentes de enfermagem durante a era COVID-19 }\end{array}$ & $\begin{array}{l}\text { Uma amostra proposital de } 10 \text { estudantes de enfermagem e } 10 \\
\text { docentes foi convidada a participar. }\end{array}$ \\
\hline $\begin{array}{c}\text { A2 } \\
\text { CINAHL }\end{array}$ & $\begin{array}{l}\text { Education Shift During COVID-19: Students' } \\
\text { Satisfaction with Emergency Distance Learning }\end{array}$ & $\begin{array}{c}\text { Sharma, Adhikari, } \\
\text { Bhattarai e Tulza } \\
(2021)\end{array}$ & $\begin{array}{l}\text { Avaliar a satisfação dos alunos com o ensino à distância de } \\
\text { emergência }\end{array}$ & $\begin{array}{l}\text { De } 336 \text { alunos de enfermagem, } 200 \text { responderam ao questionário e } \\
\text { foram adicionados ao estudo. }\end{array}$ \\
\hline $\begin{array}{c}\text { A3 } \\
\text { CINAHL }\end{array}$ & $\begin{array}{l}\text { Nursing students' experiences of a pedagogical } \\
\text { transition from campus learning to distance } \\
\text { learning using digital tools }\end{array}$ & $\begin{array}{l}\text { Langegård, Kiani, } \\
\text { Nielsen e } \\
\text { Svensson (2021) }\end{array}$ & $\begin{array}{l}\text { Descrever e avaliar as experiências de estudantes de } \\
\text { enfermagem na transição pedagógica do ensino presencial } \\
\text { tradicional para o ensino a distância por meio de ferramentas } \\
\text { digitais. }\end{array}$ & $\begin{array}{l}\text { Incluiu estudantes de enfermagem que participaram do segundo } \\
\text { semestre durante a primavera de } 2020 \text {. }\end{array}$ \\
\hline $\begin{array}{c}\text { A4 } \\
\text { CINAHL }\end{array}$ & $\begin{array}{l}\text { Nursing student perceptions of required online } \\
\text { educational programs utilized outside the classroom }\end{array}$ & $\begin{array}{l}\text { Schuler, Tyo e } \\
\text { Barnett (2021) }\end{array}$ & $\begin{array}{l}\text { Examinar as percepções dos alunos sobre programas } \\
\text { educacionais online atribuídos ao corpo docente e utilizados } \\
\text { fora da sala de aula. }\end{array}$ & Um total de 211 estudantes de enfermagem participaram do estudo. \\
\hline $\begin{array}{c}\text { A5 } \\
\text { CINAHL }\end{array}$ & $\begin{array}{l}\text { Experiences of nursing students under the } \\
\text { unprecedented abrupt online learning format forced } \\
\text { by the national curfew due to COVID-19: A } \\
\text { qualitative research study }\end{array}$ & $\begin{array}{l}\text { Suliman, Abu- } \\
\text { Moghli, Khalaf, } \\
\text { Zumot e Nabolsi } \\
\quad \text { (2021) }\end{array}$ & $\begin{array}{l}\text { Explorar e compreender a primeira experiência de estudantes de } \\
\text { graduação em enfermagem da Jordânia com OL, quando o } \\
\text { sistema educacional na Jordânia teve que mudar abruptamente } \\
\text { para o aprendizado à distância por causa da pandemia COVID- } \\
19 .\end{array}$ & $\begin{array}{l}\text { Os participantes do estudo foram } 18 \text { alunos ( } 10 \text { alunos estudavam na } \\
\text { universidade pública e } 8 \text { alunos na universidade privada). Os alunos } \\
\text { incluíram } 14 \text { mulheres e quatro homens com idades entre } 19 \text { e } 23 \\
\text { anos }\end{array}$ \\
\hline $\begin{array}{c}\text { A6 } \\
\text { PUBMED }\end{array}$ & $\begin{array}{l}\text { Nursing student experiences of remote learning } \\
\text { during the COVID-19 pandemic }\end{array}$ & $\begin{array}{l}\text { Wallace, Schuler, } \\
\text { Kaulback, Hunt e } \\
\text { Baker (2021) }\end{array}$ & $\begin{array}{l}\text { Explorar as experiências dos alunos do pré-licenciamento do } \\
\text { bacharelado em enfermagem na transição para a aprendizagem } \\
\text { remota durante o semestre da primavera de } 2020 \text { em um dos } \\
\text { primeiros estados afetados por essa transição. }\end{array}$ & $\begin{array}{l}\text { Onze alunos participaram deste estudo; } 45,45 \%(\mathrm{n}=5) \text { eram alunos } \\
\text { do terceiro ano e } 54,55 \%(\mathrm{n}=6) \text { eram alunos do último ano de } \\
\text { enfermagem. A maioria dos participantes era do sexo feminino ( } \mathrm{n}= \\
\text { 10), com média de idade de } 24,6 \text { anos. }\end{array}$ \\
\hline $\begin{array}{c}\text { A7 } \\
\text { PUBMED }\end{array}$ & $\begin{array}{l}\text { Nursing students' attitude on the practice of e- } \\
\text { learning: A cross-sectional survey amid COVID-19 } \\
\text { in Nepal }\end{array}$ & $\begin{array}{l}\text { Thapa, Bhandari e } \\
\text { Pathak (2021) }\end{array}$ & $\begin{array}{l}\text { Identificar a atitude de estudantes de enfermagem frente à } \\
\text { prática do e-learning em meio ao COVID-19. }\end{array}$ & Estudantes de enfermagem, com amostra de 470. \\
\hline
\end{tabular}

Fonte: Artigos pesquisados (2021). 


\section{Entraves na utilização do ensino remoto para os acadêmicos de enfermagem durante a pandemia de COVID-19}

O distanciamento social foi instituído devido à pandemia induzida pelo Coronavírus que, consequentemente, interrompeu o ensino convencional em sala de aula. Para enfrentar esse desafio, escolas e universidades instituíram o ensino à distância de emergência (Sharma et al., 2021). Dessa forma, a COVID-19 não só desafiou o sistema de saúde, mas também impactou a educação de enfermagem.

Portanto, diferentes métodos de ensino foram usados e com eles diversas dificuldades e impactos acadêmicos foram relatados. Foram identificados entraves que englobam a categoria de inadequação, integridade acadêmica, ambiente de aprendizagem e sobrecarga familiar, conforme demonstrado no Quadro 4.

Entre os desafios de inadequação evidenciou-se a falta de ambiente de aprendizagem em casa (Bdair, 2021; Suliman et al., 2021; Wallace et al., 2021); O uso de diferentes plataformas para ministrar, cursos, aulas ou palestras (Bdair, 2021; Langegård et al., 2021; Schuler et al., 2021); Falta de treinamento no uso de aprendizagem virtual em sala de aula (Bdair, 2021; Langegård et al., 2021; Suliman et al., 2021); Os professores não têm proficiência no uso das plataformas de software (Langegård et al., 2021; Wallace et al., 2021); Os alunos não têm proficiência no uso das plataformas de software (Bdair, 2021; Sharma et al., 2021; Suliman et al., 2021); Dificuldades de avaliação do aluno; Falta de controle sobre os parâmetros de avaliação (exames, questionários e trabalhos), etc. (Bdair, 2021).

O uso de diferentes plataformas digitais para ministrar aulas foi apontado como desafio, pois segundo os estudantes de enfermagem, os professores usavam diferentes aplicativos online para ministrar palestras devido à falta de uma plataforma padrão e eles enfrentavam um desafio para dominar todos esses aplicativos (Bdair, 2021; Langegård et al., 2021; Schuler et al., 2021)

Como esperado, dos estudos que analisaram os desafios de inadequação, a cobertura de Internet inadequada e perda de conectividade foi o desafio mais relatados pelos autores (Bdair, 2021; Sharma et al., 2021; Langegård et al., 2021; Schuler et al., 2021; Suliman et al., 2021; Wallace et al., 2021; Thapa et al., 2021). A falta de interação dos alunos também foi observada como desafio no aprendizado. Onde os alunos descrevem o sentimento de isolamento, solidão, falta de grupos de estudo e uma incapacidade de fazer perguntas aos colegas (Bdair, 2021; Wallace et al., 2021; Langegård et al., 2021).

Entre os desafios que englobam a integridade acadêmica os professores e alunos mencionaram que a aprendizagem online é inadequada para ensinar competências e habilidades práticas. Segundo os entrevistados a prática de enfermagem é o cerne da profissão, sem a prática não há como dominar as competências exigidas; mesmo o conhecimento teórico precisa estar atrelado à prática, sendo um verdadeiro desafio (Bdair, 2021; Sharma et al., 2021; Schuler et al., 2021; Suliman et al., 2021).

Outros desafios de integridade acadêmica foram a falta de profissionalismo por interrupção do aluno (Bdair, 2021); Tecnofobia (medo de se desconectar principalmente durante os exames) (Bdair, 2021; Schuler et al., 2021; Suliman et al., 2021) e a falta de comunicação da linguagem corporal e contato visual entre professores e alunos (Bdair, 2021; Langegård et al., 2021; Suliman et al., 2021; Wallace et al. 2021).

Dos desafios que englobavam o ambiente de aprendizagem evidenciou-se a falta de regras e diretrizes do processo de aprendizagem online (Bdair, 2021); Sobrecarga de atribuições (grande quantidade de tarefas) (Bdair, 2021; Suliman et al., 2021); Despreparo para a mudança abrupta para o aprendizado online; Distração por aplicativos e programas da Internet; e não é apropriado para pessoas com problemas de saúde que não conseguem sentar-se por muito tempo na frente do computador (Bdair, 2021).

E por fim, dos desafios sobre a sobrecarga familiar observou-se gastos financeiros para comprar um novo dispositivo tecnológico (Bdair, 2021; Sharma et al., 2021; Suliman et al., 2021); e obrigação doméstica para trabalhadoras e donas de casa, 
alunos e professores, principalmente para os país que tiveram que cuidar de seus filhos (Bdair, 2021; Sharma et al., 2021; Wallace et al., 2021).

Nesse contexto, esses desafios incluíram infraestrutura de tecnologia, suporte de tecnologia, espaço de aprendizagem, família e trabalho. Demonstrando que o ensino online representa desafios em diferentes contextos.

Em resposta ao início da pandemia COVID-19, os alunos enfrentaram responsabilidades crescentes fora de suas cargas de trabalho acadêmicas, o que resultou em estresse e tensão. Dos estudos que analisaram os impactos emocionais causados pelo ensino a distância em estudantes de enfermagem observou-se desmotivação (Bdair, 2021; Langegård et al., 2021); Fadiga associada à sobrecarga de tecnologia, estresse e exaustão mental (Schuler et al., 2021); medo e preocupação (medo de perder o semestre e atrasar a formatura); impotência, ansiedade (Suliman et al., 2021); isolamento e solidão (Thapa et al., 2021).

Quadro 4. Descrição dos principais entraves enfrentados por estudantes de enfermagem durante a pandemia de COVID-19. Caxias, MA, Brasil, 2021.

\begin{tabular}{|c|c|}
\hline Autor e ano & Entraves \\
\hline \multicolumn{2}{|l|}{ Inadequação } \\
\hline Bdair (2021) & 1. Falta de controle sobre os parâmetros de avaliação (exames, questionários e trabalhos). \\
\hline Bdair (2021) & 3. Dificuldades de avaliação do aluno \\
\hline $\begin{array}{l}\text { Bdair (2021), Sharma et al. (2021), } \\
\text { Langegård et al. (2021), Schuler et al. } \\
\text { (2021), Suliman et al. (2021), Wallace et } \\
\text { al. (2021) e Thapa et al. (2021) }\end{array}$ & 4. Cobertura de Internet inadequada e perda de conectividade \\
\hline $\begin{array}{l}\text { Bdair (2021), Sharma et al. (2021) e } \\
\text { Suliman et al. (2021) }\end{array}$ & 5. Os alunos não têm proficiência no uso das plataformas de software. \\
\hline $\begin{array}{l}\text { Langegård et al. (2021) e Wallace et al. } \\
\text { (2021) }\end{array}$ & 6. Os professores não têm proficiência no uso das plataformas de software. \\
\hline Bdair (2021) e Wallace et al. (2021) & 7. Falta de interação dos alunos \\
\hline $\begin{array}{l}\text { Bdair (2021), Langegård et al. (2021) e } \\
\text { Suliman et al. (2021) }\end{array}$ & 8. Falta de treinamento no uso de aprendizagem virtual em sala de aula. \\
\hline $\begin{array}{l}\text { Bdair (2021), Langegård et al. (2021) e } \\
\text { Schuler et al. (2021) }\end{array}$ & 9. Utilizando diferentes plataformas para ministrar, cursos, aulas ou palestras. \\
\hline \multicolumn{2}{|l|}{ Integridade Acadêmica } \\
\hline $\begin{array}{l}\text { Bdair (2021), Sharma et al. (2021), } \\
\text { Schuler et al. (2021) e Suliman et al. } \\
\text { (2021) }\end{array}$ & 10. Inapropriado para ensinar competências práticas \\
\hline $\begin{array}{l}\text { Bdair (2021), Langegård et al. (2021) e } \\
\text { Suliman et al. (2021), Wallace et al. } \\
\text { (2021) }\end{array}$ & 12. Falta de comunicação da linguagem corporal e contato visual entre professores e alunos \\
\hline \multicolumn{2}{|l|}{ Ambiente de aprendizagem } \\
\hline $\begin{array}{l}\text { Bdair (2021), Schuler et al. (2021) e } \\
\text { Suliman et al. (2021) }\end{array}$ & 13. Tecnofobia (medo de se desconectar principalmente durante os exames). \\
\hline Bdair (2021) & 14. Falta de regras e diretrizes do processo de aprendizagem online \\
\hline
\end{tabular}


Bdair (2021) e Suliman et al. (2021)

Bdair (2021)

Bdair (2021)

Bdair (2021)
15. Sobrecarga de atribuições (grande quantidade de tarefas)

16. Despreparo para a mudança abrupta para o aprendizado online.

17. Distração por aplicativos e programas da Internet.

18. Não é apropriado para pessoas com problemas de saúde que não conseguem sentar-se por muito tempo na frente do computador

\section{Sobrecarga Familiar}

Bdair (2021), Sharma et al. (2021) e Suliman et al. (2021)

Bdair (2021), Suliman et al. (2021) e Wallace et al. (2021)

19. Gasto financeiro para comprar um novo dispositivo tecnológico para família

20. Obrigação doméstica para trabalhadoras e donas de casa, alunos e professores (ex. cuidar dos filhos).

Fonte: Artigos Analisados (2021).

\section{Benefícios advindos do ensino remoto para os acadêmicos frente ao contexto de pandemia}

O aprendizado online foi uma importante estratégia alternativa de ensino durante o distanciamento social do COVID19. Os benefícios na aquisição de conhecimentos da aprendizagem online foram descritos em cinco das evidências analisadas, essas informações podem ser consultadas no quadro 5 .

Nos estudos, destaca-se que a principal vantagem do aprendizado online reside na flexibilidade, onde pode ser conduzido em um momento conveniente. Isso ajuda a garantir aprendizado remoto onde quer que haja acesso à internet, fazendo com que os alunos economizem tempo e recursos por não usarem transporte e o tempo gasto no percurso até a instituição de ensino (Bdair, 2021; Schuler et al., 2021; Suliman et al., 2021; Walacce et al., 2021; Thapa et al., 2021).

O tempo extra de estudo obtido por não ter longos deslocamentos permite se dedicar um tempo para o autocuidado (Walacce et al., 2021). A flexibilidade permite o acesso a aplicativos amigáveis ao usuário (Bdair, 2021; Schuler et al., 2021); o acesso a palestras em horários convenientes; e melhora a comunicação entre alunos e professores (Bdair, 2021).

O uso do ensino remoto durante o COVID-19 melhorou as habilidades de aprendizado online dos alunos, o que apoia a adoção futura dessa forma de aprendizado. $\mathrm{O}$ ambiente de aprendizagem online é controlado pelo professor, evitando qualquer possível interrupção do aluno. Enquanto isso, se os alunos tiverem dúvidas ou não tiverem certeza sobre um tópico ou conceito, eles podem levantar a mão e pedir permissão para participar. Isso ajuda a criar um ambiente de aprendizagem mais adequado para uma melhor compreensão (Bdair, 2021).

Os alunos relataram que alguns dos programas aumentaram seu aprendizado e capacidade de pensar criticamente. No nível educacional, os alunos explicaram que ensino remoto era obrigatório e não fácil; no entanto, foi uma experiência agradável porque incentivou a autossuficiência e os motivou a desenvolver suas habilidades de raciocínio e resolução de problemas. O Ensino Remoto foi uma oportunidade de aprender como gerenciar as necessidades de aprendizagem de forma eficaz. Nesse contexto, os estudos mostram em síntese que o ensino remoto melhora o desempenho acadêmico dos alunos; melhora suas habilidades de aprendizagem; e permite a conexão a uma ampla variedade de recursos de aprendizagem pela internet, permitindo sua conquista acadêmica (Bdair, 2021; Schuler et al., 2021; Suliman et al., 2021; Walacce et al., 2021; Thapa et al., 2021).

Entretanto, na fala dos entrevistados o ensino remoto é inadequado para ensinar competências e habilidades práticas (Bdair, 2021; Sharma et al., 2021; Schuler et al., 2021; Suliman et al., 2021). Dessa forma, subentende-se que o ensino traz benefícios para aquisição de conhecimentos teóricos, sendo necessário, associar a prática com o ensino presencial, sendo está uma das principais barreiras enfrentadas pelas instituições durante a pandemia de COVID-19. 
Com relação a vantagem da aprendizagem centrada no aluno, segundo Bdair (2021) o aluno é sempre o centro do processo de aprendizagem. A adoção de uma estratégia de aprendizagem online permite que os alunos se tornem aprendizes autônomos. Corroboram com essa afirmativa Schuler et al. (2021), Suliman et al. (2021), Walacce et al. (2021) e Thapa et al. (2021).

Quadro 5. Benefícios advindos do ensino remoto para os acadêmicos frente ao contexto de pandemia. Caxias, MA, Brasil, 2021.

\begin{tabular}{|c|c|}
\hline Autor e ano & Benefícios \\
\hline \multicolumn{2}{|l|}{ Ambiente de aprendizagem flexível } \\
\hline Bdair (2021), Schuler et al. (2021) & 1. Aplicativos amigáveis ao usuário. \\
\hline Bdair (2021) & 2. Palestras em horários convenientes. \\
\hline $\begin{array}{l}\text { Bdair (2021), Schuler et al. (2021), Thapa et } \\
\text { al. (2021) }\end{array}$ & 3. Estratégia inovadora com utilização de grande variedade de recursos. \\
\hline $\begin{array}{l}\text { Bdair (2021), Schuler et al. (2021), Suliman } \\
\text { et al. (2021) }\end{array}$ & 4. Manter a continuidade do ensino. \\
\hline $\begin{array}{l}\text { Bdair (2021), Schuler et al. (2021), Suliman } \\
\text { et al. (2021), Walacce et al. (2021), Thapa } \\
\text { et al. (2021) }\end{array}$ & 5. Economize tempo e recursos. \\
\hline Bdair (2021) & 6. Comunicação fácil com professores. \\
\hline Bdair (2021), Thapa et al. (2021) & 7. Apropriado para alunos trabalhadores. \\
\hline \multicolumn{2}{|l|}{ Conquista acadêmica } \\
\hline $\begin{array}{l}\text { Bdair (2021), Schuler et al. (2021), Suliman } \\
\text { et al. (2021), Walacce et al. (2021), Thapa } \\
\text { et al. (2021) }\end{array}$ & 8. Melhorar o desempenho acadêmico dos alunos. \\
\hline $\begin{array}{l}\text { Bdair (2021), Schuler et al. (2021), Thapa et } \\
\text { al. (2021) }\end{array}$ & 9. Conexão a uma ampla variedade de recursos de aprendizagem pela Internet. \\
\hline $\begin{array}{l}\text { Bdair (2021), Schuler et al. (2021), Suliman } \\
\text { et al. (2021), Walacce et al. (2021), Thapa } \\
\text { et al. (2021) }\end{array}$ & 10. Melhorar as habilidades de aprendizagem online dos alunos. \\
\hline \multicolumn{2}{|l|}{ Aprendizagem centrada no aluno } \\
\hline $\begin{array}{l}\text { Bdair (2021), Schuler et al. (2021), Suliman } \\
\text { et al. (2021), Walacce et al. (2021) }\end{array}$ & 11. Os alunos tornam-se aprendizes autônomos e motivados. \\
\hline $\begin{array}{l}\text { Bdair (2021), Schuler et al. (2021), Suliman } \\
\text { et al. (2021), Walacce et al. (2021), Thapa } \\
\text { et al. (2021) }\end{array}$ & 12. Capacidade de revisar aulas gravadas muitas vezes ou aulas perdidas \\
\hline
\end{tabular}

Fonte: Artigos Analisados (2021).

\section{Recursos tecnológicos utilizados durante as aulas de ensino remoto}

Nos dias atuais, o uso da tecnologia da informação (TI) na educação é indiscutível. O crescente avanço da TI mudou o cenário da educação. Com o surgimento da situação atual do COVID-19, ela sem dúvida forneceu uma solução para a maioria de nossas necessidades educacionais, quando todas as instituições de ensino permaneceram fechadas devido à pandemia (Thapa et al., 2021). A tecnologia fornece uma plataforma para que os alunos considerem e trabalhem através de cenários clínicos complexos que eles não encontrariam em seus estágios clínicos, ajudando assim a preencher a lacuna entre a teoria e a prática e aumentar as habilidades de raciocínio clínico (Schuler et al., 2021). 
Research, Society and Development, v. 11, n. 1, e44911124883, 2022

(CC BY 4.0) | ISSN 2525-3409 | DOI: http://dx.doi.org/10.33448/rsd-v11i1.24883

Evidenciou-se que entre os recursos tecnológicos o Zoom foi software de teleconferência mais utilizado (Sharma et al., 2021; Langegård et al., 2021; Schuler et al., 2021; Suliman et al., 2021); seguido do Microsoft Teams (Sharma et al., 2021; Langegård et al., 2021; Suliman et al., 2021). Outras plataformas que podem ser utilizadas para teleconferência incluíram o Facebook; Youtube; e Skype (Suliman et al., 2021) (Quadro 6)

Como mensageiros que serviram de suporte durante o ensino remoto, observou-se o uso de E-mail; Messenger; Viber (Sharma et al., 2021); e WhatsApp (Suliman et al., 2021) (Quadro 6). Evidenciando que as redes sociais foram importantes ferramentas utilizadas por alunos e professores.

O Ambiente Virtual de Aprendizagem (AVA) foi o recurso tecnológico mais utilizado pelos professores para o desenvolvimento e distribuição de conteúdo diversos das disciplinas para alunos em geral (Bdair, 2021; Sharma et al., 2021; Langegård et al., 2021; Schuler et al., 2021; Suliman et al., 2021; Wallace et al., 2021; Thapa et al., 2021) (Quadro 6).

Nesse ambiente os alunos tinham preferência pelas aulas em vídeo pré-gravadas (Langegård et al., 2021; Schuler et al., 2021). Apenas um estudo retratou o uso de simulação, podcasts e animações (Schuler et al., 2021) (Quadro 6). Isso é justificado pelo fato dos estudos anteriores relataram resultados em relação aos benefícios e obstáculos do ensino a distância usando ferramentas digitais para a educação de enfermagem, não dando tanta ênfase em detalhes de como as aulas práticas e teóricas funcionavam, apenas retratavam quais tecnologias eram utilizadas durantes as aulas.

Dessa forma, os problemas incluem restrições tecnológicas, como a qualidade da conectividade com a Internet para permitir o acesso a recursos online e restrições financeiras. Se o ensino remoto pode ser facilitado para o usuário, a necessidade de reduzir as barreiras técnicas, complementadas com programas que podem melhorar as habilidades práticas de aprendizagem e reduzir as desigualdades de acesso à tecnologia (Bdair, 2021; Sharma et al., 2021; Langegård et al., 2021; Schuler et al., 2021; Suliman et al., 2021; Wallace et al., 2021; Thapa et al., 2021). 
Research, Society and Development, v. 11, n. 1, e44911124883, 2022

(CC BY 4.0) | ISSN 2525-3409 | DOI: http://dx.doi.org/10.33448/rsd-v11i1.24883

Quadro 6. Recursos tecnológicos utilizados durante as aulas de ensino remoto. Caxias, MA, Brasil, 2021.

\begin{tabular}{|c|c|c|c|c|c|c|c|c|c|c|c|c|c|c|}
\hline Autor ano & Zoom & $\begin{array}{c}\text { Equipe } \\
\text { Microsoft }\end{array}$ & E-mail & YT & FB & Wpp & Skype & Messenger & Viber & AVA & $\begin{array}{l}\text { Aulas em } \\
\text { vídeo pré- } \\
\text { gravadas }\end{array}$ & Podcasts & Animações & Simulação \\
\hline $\begin{array}{c}\text { Sharma et al. } \\
\text { (2021) }\end{array}$ & $\mathrm{X}$ & $\mathrm{X}$ & $\mathrm{X}$ & - & - & - & - & $\mathrm{X}$ & $\mathrm{X}$ & $\mathrm{X}$ & - & - & - & - \\
\hline $\begin{array}{c}\text { Langegård et al. } \\
\text { (2021) }\end{array}$ & $\mathrm{X}$ & $\mathrm{X}$ & - & - & - & - & - & - & - & - & $\mathrm{X}$ & - & - & - \\
\hline Bdair (2021) & - & - & - & - & - & - & - & - & - & $\mathrm{X}$ & - & - & - & - \\
\hline $\begin{array}{l}\text { Schuler et al. } \\
\text { (2021) }\end{array}$ & $\mathrm{X}$ & - & - & - & - & - & - & - & - & - & $\mathrm{X}$ & $\mathrm{X}$ & $\mathrm{X}$ & $X$ \\
\hline $\begin{array}{l}\text { Suliman et al. } \\
\text { (2021) }\end{array}$ & $X$ & $\mathrm{X}$ & - & $\mathrm{X}$ & $\mathrm{X}$ & $\mathrm{X}$ & $X$ & - & - & - & - & - & - & - \\
\hline $\begin{array}{l}\text { Wallace et al. } \\
\text { (2021) }\end{array}$ & & & & & & & & & & $\mathrm{X}$ & - & - & - & - \\
\hline $\begin{array}{l}\text { Thapa et al. } \\
\text { (2021) }\end{array}$ & - & - & - & - & - & & - & - & - & $\mathrm{X}$ & - & - & - & - \\
\hline Total & 04 & 03 & 01 & 01 & 01 & 01 & 01 & 01 & 01 & 05 & 02 & 01 & 01 & 01 \\
\hline
\end{tabular}

Legenda: Hífen (-) = não reportado; YT = Youtube; FB = Facebook; Wpp = WhatsApp; AVA = Ambiente Virtual de Aprendizagem. Fonte: Artigos Analisados (2021). 


\section{Discussão}

\section{Pandemia de COVID-19 e os desafios do ensino remoto para acadêmicos de enfermagem}

Como reflexo da pandemia de COVID-19 enfrentada em todo o mundo, o ensino da enfermagem nas diversas instituições, assim como a educação no geral, sofreu uma abrupta mudança que trouxe consequências negativas para os educandos.

Um dos principais desafios do ensino remoto está na dependência de internet para que ele seja realizado. Pesquisa feita por Sharma et al. (2021) em Nepal com 200 estudantes de enfermagem demonstrou que 87\% dos participantes relataram a perda de conectividade de internet como principal obstáculo para a sua aprendizagem. Corroborando com esse resultado, o estudo de Thapa et al. (2021) com estudantes de enfermagem, também relatou que a maioria $(81,7 \%)$ dos entrevistados citou os problemas com a internet como a principal desvantagem do ensino remoto, além da parte financeira para comprar novos dispositivos tecnológicos ser um problema para algumas famílias.

Observou-se que nos artigos analisados, a maioria dos alunos teve problemas de internet e problemas tecnológicos, sendo a internet um meio tecnológico vital para o funcionamento desses softwares, uma vez que, quase todos esses programas só funcionam no ambiente online, afetando a educação de alunos com baixo poder aquisitivo para o contrato de internet de qualidade, assinatura de internet e até mesmo para aquisição de dispositivos tecnológicos.

Mesmo que seja adotada a educação a distância em algumas situações e sejam utilizadas estratégias como simulações e estudos de caso, por ser um curso clínico/prático, a enfermagem exige a prática, de modo presencial com pacientes, para que os alunos consigam adquirir as habilidades necessárias (Sharma et al., 2021; Suliman et al., 2021). Estudo brasileiro feito na Bahia corrobora com os achados da pesquisa ao citar a inviabilidade de realização das aulas práticas como entrave do ensino remoto para os acadêmicos de enfermagem (Silva et al., 2021). Ademais, o ensino online não permite que o educando trabalhe sua interação em equipe, essencial para a profissão.

Sobre a falta de interação, Thapa et al. (2021) apontam que é inviável e dificultoso gerenciar com e-learning a clínica de enfermagem, pois é necessário que os acadêmicos aprendam por meio do atendimento de pacientes reais. Semelhante a isso, estudo realizado por Langegård et al. (2021) na Suécia com 96 alunos de enfermagem relata a falta de interação dos alunos entre si e com professores como um desafio da educação a distância, o que impede que eles tirem suas dúvidas e desenvolvam seu potencial ao máximo.

Como a maioria dos alunos realiza o estudo remoto em casa, o gerenciamento do tempo entre assistir as aulas online, estudar, realizar as atividades de casa e cuidar dos filhos é uma dificuldade a ser enfrentada. Muitos são casados, tem a familia numerosa, possuem filhos e as vezes não podem contar com a assistência de seus parceiros, assim, todos esses aspectos tornam a concentração em seus estudos difícil. A situação piora para aqueles que trabalham, principalmente em hospitais, e não podem tirar folga para estudar ou escolhem trabalhar em turnos noturnos para estudar durante o dia, o que acaba gerando maior cansaço e estresse sobre eles (Suliman et al., 2021).

Os entraves incluem ainda a falta de habilidade dos professores e dos próprios alunos para utilizar as diversas plataformas online e a grande quantidade de tarefas passadas a eles a fim de recompensar a falta das aulas presenciais. Dessa forma, tais fatores contribuem para o surgimento de ansiedade e a fadiga digital relacionada ao número aplicativos e programas educacionais online e a quantidade de tempo gasto em frente aos dispositivos tecnológicos (Schuler et al., 2021). 


\section{Principais vantagens do ensino remoto para os acadêmicos de enfermagem no contexto de pandemia}

Mesmo com os diversos desafios enfrentados, o ensino remoto também trouxe alguns benefícios. Entre eles está a sua flexibilidade, na qual, em sua pesquisa realizada na Arábia Saudita, Bdair (2021) relata que o estudante pode fazer seu próprio horário de estudo, no ambiente que ele se sentir mais confortável, além disso, não necessita se locomover. Corroborando, Suliman et al. (2021) afirmam que, no nível socioeconômico, os estudantes economizam dinheiro por não precisar gastar com transporte e outras despesas para viajar até o campus de sua faculdade, podendo também passar mais tempo com a família.

Outra vantagem abordada por alguns estudos é a melhora do aprendizado e do pensamento crítico. Com a educação online os acadêmicos se tornam aprendizes autodirigidos, responsáveis por buscar conteúdos e escolher a melhor maneira como desejam aprender. $\mathrm{O}$ aprendizado online permite também que os alunos revisem as aulas gravadas muitas vezes e oferece diversos sites de aprendizado na Internet, aumentando sua capacidade de aprender e resolver problemas (Bdair, 2021; Schuler et al., 2021).

Desse modo, segundo Vieira e Santos (2020) a Metodologia Ativa utilizada no ensino remoto tem como objetivo desenvolver a autonomia individual dos discentes, estimulando sua compreensão de aspectos socioeconômicos, cognitivos, afetivos, culturais e políticos.

\section{Ensino remoto e principais recursos tecnológicos utilizados}

Em decorrência da Pandemia de COVID-19 mudanças imediatas tiveram que ser adotadas na educação, desta forma a modalidade de ensino em decorrência das circunstâncias passaram a ser totalmente remotas (Wallace et al., 2021). Nesse contexto Branco (2020) relatam que as tecnologias foram consideradas ferramentas alternativas e imprescindíveis durante a pandemia da COVID-19, pois com a rápida disseminação do vírus e o risco de contágio a suspensão das aulas presenciais foram uma das condutas adotadas e que influenciou diretamente no planejamento e rotina acadêmica de professores e estudantes em todo mundo.

Dentre os recursos tecnológicos mais adotados pelas instituições de ensino superior no âmbito de ensino da enfermagem destacou-se a plataforma Zoom (Wallace et al., 2021; Thapa et al., 2021; Suliman, 2021; Schuler et al., 2021; Langegård et al., 2021).

Segundo Santos e Monteiro (2020) o Zoom é um aplicativo prático para realizar ou participar de reuniões em vídeo, podendo ser acessado de diferentes dispositivos móveis com sistemas operacionais variados, sendo possível convidar os participantes por e-mail, SMS e até mesmo pelas redes sociais, ainda possibilita o compartilhamento de arquivos, textos que favorece a apresentação dos conteúdos pelos professores, e a interface do software possibilita que todos os participantes apareçam na tela, lado a lado, como se estivessem em sala de aula.

Entretanto para Santos (2020) ressalta em seu estudo que reuniões via Zoom exigem mais atenção, resultando em um esforço psicológico mais intenso e que em muitos casos pode levar ao esgotamento, principalmente quando algum componente da comunicação está ausente ou limitado, fazendo com que o emissor e receptor tenham que fazer mais esforços para prestar atenção, se expressar e entender corretamente um ao outro, e para aqueles que não estão acostumados possa aumentar os riscos dos níveis de stress e exaustão emocional e um "empobrecimento" da troca direta de experiência entre professor e aluno, dificultando o processo de aprendizagem.

O ambiente virtual Google classroom juntamente utilizado com o google meet também foi uma das plataformas mais utilizadas pelas instituições para as aulas remotas, e apresenta funcionalidades semelhantes ao zoom (Thapa et al., 2021; Sharma et al., 2021; Suliman et al., 2021). 
De acordo com Santos e Monteiro (2020) o Google classroom foi a plataforma mais escolhida para mediação remota, pois além de ser uma ferramenta online, não necessita de instalação local ou um servidor exclusivo, e ainda comporta alunos e professores, facilitando a entrada (login), um outro destaque é que a plataforma possui integração de diferentes recursos que são disponibilizados pelo próprio Google como: Hangout, Gmail, Google Drive, Google Docs e Google Forms, além disse facilita para o professor programar aulas e atividades e proporciona liberdade para que o aluno acesse o conteúdo de qualquer lugar.

Esses achados corroboram com os estudos de Alves (2020) que relata que o Google classroom é a plataforma mais utilizada para as aulas remotas desde a mudança de modalidade de ensino, tanto em instituições de ensino básico como também nas instituições de ensino superior públicas.

Outros recursos utilizados em menor frequência do que o zoom e o Google classroom foram o Microsoft teams, Email, Viber, Mensseger (Langegård et al., 2021; Sharma et al., 2021; Thapa et al., 2021). Tais recursos também foram identificados nas pesquisas de Miranda, Lima, Oliveira e Telles (2020) que além desses constataram o uso de Moodle, chats, lives e softwares livres para edição e gravação de videoaulas.

Observa-se que os recursos utilizados são ferramentas que permitem uma flexibilidade e usabilidade de acesso, tendo boa aceitação no geral, entretanto para que sejam efetivas em suas totalidades é necessário considerar outros aspectos como acesso à internet e a aparelhos tecnológicos como celulares e computadores para que todos possam ter acesso e participação, reduzindo assim as desigualdades (Santos \& Monteiro 2020).

\section{Principais estratégias para facilitar a adesão ao ensino remoto}

A pandemia trouxe consigo uma ampla gama de mudanças em todos os setores da sociedade, portanto, com a educação não foi diferente. O processo de ensino-aprendizagem das instituições teve que ser adaptado ao ensino remoto e neste sentido, a resiliência foi uma aliada para quebrar barreiras e possibilitar a continuidade dos cursos (Wallace et al., 2021). Minimizando ainda, o risco de desenvolver transtornos psicológicos (Ferreira et al., 2021).

Neste sentido, é importante destacar que apesar das dificuldades enfrentadas no ensino remoto algumas estratégias podem facilitar o processo de aprendizagem, onde Thapa et al. (2021) afirmam que, a oferta de internet de qualidade e instruções para resolver problemas advindos do uso de novas tecnologias tornam o e-learning (ensino não presencial apoiado por tecnologias da informação), muito útil e proveitoso para os acadêmicos. Corroborando com a afirmativa, Suliman et al. (2021) discorrem que o ensino remoto tem sido muito útil no contexto da pandemia, porém, deve-se realizar mais estudos para avaliar a eficácia e a melhor forma de utilizar o e-learning.

Em sintonia com o exposto, o estudo de Langegård et al. (2021) avalia que a melhor estratégia para a utilização do ensino remoto seria a aplicação de um ensino misto que mesclasse aulas presenciais e virtuais, promovendo maior interação entre os alunos, ampliando a gama de conteúdos ofertados e rompendo as barreiras impostas pelos estudantes quanto as aulas online. Neste ponto, percebe-se que a utilização de aulas online já vem sendo utilizadas por diversas universidades em todo o mundo, de maneira complementar ao ensino presencial (Xavier et al., 2020).

Outras estratégias para melhorar a qualidade do ensino remoto incluem: colaboração entre professores para aperfeiçoar o ensino-aprendizagem de conteúdos inter-relacionados, evitar desperdício de tempo e distrações e redundâncias (Schuler et al., 2021). Merece destaque ainda, a escolha de um ambiente adequado para estudar e aparelhos eletrônicos em boas condições de uso (Pereira et al., 2021).

É notado que, para haver maior qualidade e otimizar o e-learning, é necessário que haja treinamentos para, auxiliar professores e discentes a utilizar as tecnologias e ferramentas ofertadas por cada instituição de ensino e promover 
conhecimento geral sobre as tecnologias disponíveis; deve-se realizar pequenas avaliações frequentes para verificar o grau de aprendizado dos alunos; realizar monitoramento da qualidade do ensino, utilizando aplicativos que realizem as avaliações sem consultas a outras páginas online e realização de testes orais e; utilização de técnicas voltadas a evitar distrações durante as aulas (Bdair, 2021).

Corroborando com a afirmativa, Sharma et al. (2021) destaca a necessidade de capacitação dos docentes para atuar e potencializar o ensino remoto, e a criação de e-learning próprios de cada instituição para facilitar o aprendizado dos acadêmicos.

\section{Considerações Finais}

Este estudo permitiu compreender os entraves e benefícios que permeiam a utilização do ensino remoto para os acadêmicos de enfermagem no que se refere ao contexto da pandemia. Neste sentido, foi observado que a pandemia afetou a forma como o conhecimento é repassado, e dificultou a realização de aulas práticas para os alunos onde, o ensino remoto se tornou a alternativa mais segura e eficaz, utilizando diversas plataformas online, como Zoom, Google Meet e Teams.

Porém, há certa resistência para a adesão ao e-learning, neste ponto, as estratégias para melhorar a qualidade do ensino remoto se destacam por buscar aprimorar a forma de estudar, e estimular os acadêmicos a expandir e aprofundar as áreas de conhecimento, onde a realização de aulas mistas e treinamentos para o manuseio das tecnologias por alunos e professores, além de técnicas para evitar distrações, entre outras estratégias, podem romper barreiras e consolidar a utilização do ensino remoto.

É fundamental destacar que, a graduação em enfermagem é um curso que agrega teoria e prática para envolver os estudantes no processo de aprendizagem, portanto, o ensino remoto deve se fazer como forma não a substituir o conhecimento prático, e sim, possibilitar que os alunos englobem mais conhecimento.

Como limitações do estudo tem-se o fato da escassez de literatura, onde os artigos, muitas vezes, não detalharam como se fez o uso das novas tecnologias no ensino remoto, somente citando os nomes de plataformas utilizadas. Além disso, muitos estudos não estavam disponíveis na integra e a maior parte das pesquisas possuía abordagem qualitativa, o que permite apenas uma análise subjetiva e dificulta a generalização dos resultados.

Por fim, o estudo possibilitou expandir os conhecimentos acerca da pandemia de COVID-19, as mudanças na sociedade ocasionadas pela patologia, e os desafios e vantagens do ensino remoto durante esse período. Dessa forma, considera-se que novas pesquisas devem ser realizadas nesta linha de investigação, a fim de provar a importância e os benefícios do e-learning para o processo de aprendizagem.

\section{Referências}

Alves, L. (2020). Educação remota: entre a ilusão e a realidade. Interfaces Científicas, 8(3), 348-365.

Bdair, I. A. (2021). Nursing students' and faculty members' perspectives about online learning during COVID-19 pandemic: A qualitative study. Teaching and Learning in Nursing, 16, 220-226.

Bork, A. M. T. (2005). Enfermagem baseada em evidencias. Guanabara Koonga.

Branco, E. P. (2020). Recursos tecnológicos e os desafios da educação em tempos de pandemia. CIET-ENPED - Congresso Internacional de educação e Tecnologia, São Paulo, SP, Brasil, 5. Recuperado de https://cietenped.ufscar.br/submissao/index.php/2020/article/view/1736.

Bueno, I., Bueno, M. O. B., \& Maciel, R. F. (2020). A utilização da tecnologia em tempos de pandemia. Cad. Ens. Ling. Tecno., 1(2), $223-234$.

Ferreira, D. A. S., Melo, K. C., Miranda, L. S. C., Oliveira, A. T. F., Hernandes, L. F., Bomfim, D. M. M., \& Oliveira, S. A. (2021). Predisposição dos transtornos ansiosos em profissionais de saúde: uma revisão integrativa. Research, Society and Development, 10(13), 1-14. 
Research, Society and Development, v. 11, n. 1, e44911124883, 2022

(CC BY 4.0) | ISSN 2525-3409 | DOI: http://dx.doi.org/10.33448/rsd-v11i1.24883

Gusso, H. L., Archer, A. B., Luiz, F. B., Sahão, F. T., Luca, G. G., Henklain, M. H. O., \& Gonçalves, V. M. (2020). Ensino superior em tempos de pandemia: diretrizes à gestão universitária. Educação \& Sociedade, 41, 1-27.

Langegård, U., Kiani, K. Nielsen, S. J., \& Svensson, P. (2021). Nursing students' experiences of a pedagogical transition from campus learning to distance learning using digital tools. BMC Nursing, 20(23), 1-10.

Magalhães, A. J. A., Rocha, M. H. A., Santos, S. C., Dantas, C. B., Manso, G. J. M. C., \& Ferreira, M. D. A. (2020). O ensino da anamnese assistido por tecnologias digitais durante a pandemia da COVID-19 no Brasil. Revista Brasileira. Educ. Med., 44, 1-7.

Miranda, K. K. C. O., Lima, A. S., Oliveira, V. C. M., \& Telles, C. B. S. (2020, out). Aulas remotas em tempo de pandemia: desafios e percepções de professores e alunos. Conedu- Congressoa Nacional de Educação, Maceió, AL, Brasil, 7. https://editorarealize.com.br/editora/anais/conedu/2020/TRABALHO_EV140_MD1_SA_ID5382_03092020142029.pdf.

Oliveira, F. B. M., Costa, A. C. A. L., Alves, D. L., França, J. F., Macedo, M. S., \& Santos, R. D. (2016). Relação entre a sobrecarga de trabalho e erros de administração de medicação na assistência hospitalar. Revista Ciências \& Saberes, 2(2), 325-334.

Pereira, N. R., Medeiros, S. L. D. S., \& Nascimento, J. A. (2021). Mapas conceituais como estratégias no ensino remoto e os desafios dos estudantes nesta modalidade no Curso Técnico em Agropecuária integrado do IFMG-Campus Bambuí. Research, Society and Development, 10(9), 1-23.

Santos, H. M. R. (2020). Os desafios de educar através da Zoom em contexto de pandemia: investigando as experiências e perspectivas dos docentes portugueses. Práxis Educativa, 15, 1-17.

Santos, V. B., Jr., \& Monteiro, J. C. S. (2020). Educação e COVID-19: as tecnologias digitais mediando a aprendizagem em tempos de pandemia. Revista Encantar, 2, 1-15

Sarti, T. D., Lazarini, W. S., Fontenelle, L. F., \& Almeida, A. P. S. C. (2020). Qual o papel da Atenção Primária à Saúde diante da pandemia provocada pela COVID-19? Epidemiol. Serv. Saude, 29(2), 1-5.

Schuler, M. S., Tyo, M. B., \& Barnett, K. (2021). Nursing student perceptions of required online educational programs utilized outside the classroom. Nurse Education Today, 105.

Sharma, M., Adhikari, T., Bhattarai, T., \& Tulza, K. C. (2021). Education Shift During COVID-19: Students' Satisfaction with Emergency Distance Learning. International Journal of Nursing Education, 13(3).

Silva, F. O., Santos, B. M. L., Jesus, A. C. S., Silva, J. M. Q., Lefundes, T. B., \& Anjos, K. F. (2021). et al. Experiência em aulas remotas no contexto da pandemia da COVID-19. Rev. enferm. UFPE on line, 15(1), 1-17.

Suliman, W. A., Abu-Moghli, F. A., Khalaf, I., Zumot, A. F., \& Nabolsi, M. (2021). Experiences of nursing students under the unprecedented abrupt online learning format forced by the national curfew due to COVID-19: A qualitative research study. Nurse Education Today, 100, 1-6.

Thapa, P., Bhandari, S. L., \& Pathak, S. (2021). Nursing students' attitude on the practice of e-learning: A cross-sectional survey amid COVID-19 in Nepal. Nepal. PLOS ONE, 16(6).

Vieira, T. D. G. F., \& Santos, M. L. S. C. (2020). Estratégias pedagógicas e uso de metodologias ativas na graduação em Enfermagem em tempos de pandemia do Coronavírus - COVID-19. Research, Society and Development, 9(11), 1-13.

Wallace, S., Schuler, M. S., Kaulback, M., Hunt, K., \& Baker, M. (2021). Nursing student experiences of remote learning during the COVID-19 pandemic. Nursing Forum, 56(3): 612-618.

Xavier, T. B., Barbosa, G. M., Meira, C. L. S., Neto, N. C., \& Pontes, H. A. R. (2020). Utilização de Recursos Web na educação em Odontologia durante Pandemia COVID-19. Braz. J. Hea. Rev., 3(3), 4989-5000. 